\title{
Long term outcomes of transplantation using kidneys from expanded criteria donors: prospective, population based cohort study
}

\author{
Olivier Aubert,, Nassim Kamar,2,3,4,5 Dewi Vernerey,1 Denis Viglietti,, 6 Frank Martinez, \\ Jean-Paul Duong-Van-Huyen,, ${ }^{1,8}$ Dominique Eladari, ${ }^{1,9}$ Jean-Philippe Empana, ${ }^{1}$ Marion Rabant, ${ }^{8}$ \\ Jerome Verine, ${ }^{10}$ Lionel Rostaing, 2,3,4,5 Nicolas Congy,, 11,12 Céline Guilbeau-Frugier,4,13 \\ Georges Mourad,, 14 Valérie Garrigue,5,14 Emmanuel Morelon,, 15,16 Magali Giral,5, 16,17 \\ Michèle Kessler,, 16, 18 Marc Ladrière, 5, 16,18 Michel Delahousse, 1, 19 Denis Glotz,, 1, 5, 6 \\ Christophe Legendre, 1, 5, 7,16 Xavier Jouven, ${ }^{1,20}$ Carmen Lefaucheur, ${ }^{1,5,6}$ Alexandre Loupy 1, 5,7
}

For numbered affiliations see end of article.

Correspondence to: A Loupy alexandreloupy@gmail.com

Additional material is published online only. To view please visit the journal online (http://dx.doi. org/10.1136/bmj.h3557)

Cite this as: $B M J$ 2015;351:h3557 doi: $10.1136 /$ bmj.h3557

Accepted: 17 June 2015

\section{ABSTRACT}

\section{OBJECTIVES}

To assess the long term outcomes of transplantation using expanded criteria donors (ECD; donors aged $\geq 60$ years or aged 50-59 years with vascular comorbidities) and assess the main determinants of its prognosis.

DESIGN

Prospective, population based cohort study.

SETTING

Four French referral centres.

\section{PARTICIPANTS}

Consecutive patients who underwent kidney transplantation between January 2004 and January 2011, and were followed up to May 2014. A validation cohort included patients from another four referral centres in France who underwent kidney transplantation between January 2002 and December 2011.

\section{MAIN OUTCOME MEASURES}

Long term kidney allograft survival, based on systematic assessment of donor, recipient, and transplant clinical characteristics; preimplantation biopsy; and circulating levels of donor specific anti-HLA (human leucocyte antigen) antibody (DSA) at baseline.

\section{WHAT IS ALREADY KNOWN ON THIS TOPIC}

Organ transplants from expanded criteria donors (donors aged $\geq 60$ years, or aged 50-59 years with vascular comorbidities) are increasingly becoming a main resource for treating end stage renal disease

Use of ECD kidney transplants has evolved unequally worldwide, and a high proportion of these kidneys are discarded

Organ shortages urgently need the optimisation and increased efficacy of ECD transplantation

\section{WHAT THIS STUDY ADDS}

In this large scale prospective study, the main independent determinants of improved ECD outcome were the absence of donor specific anti-human leucocyte antigen antibodies in transplant recipients, and reduction of cold ischaemia time After correcting these two factors, ECD transplantation can yield satisfactory long term results, and achieve a graft survival comparable to that of transplants from standard criteria donor

Dedicated ECD specific allocation policies are mandatory to extend the life span of aged organs

\section{RESULTS}

The study included 6891 patients (2763 in the principal cohort, 4128 in the validation cohort). Of 2763 transplantations performed, 916 (33.2\%) used ECD kidneys. Overall, patients receiving ECD transplants had lower allograft survival after seven years than patients receiving transplants from standard criteria donors (SCD; $80 \%$ v 88\%, $\mathrm{P}<0.001)$. Patients receiving ECD transplants who presented with circulating DSA at the time of transplantation had worse allograft survival after seven years than patients receiving ECD kidneys without circulating DSA at transplantation ( $44 \%$ v $85 \%$, $\mathrm{P}<0.001)$. After adjusting for donor, recipient, and transplant characteristics, as well as preimplantation biopsy findings and baseline immunological parameters, the main independent determinants of long term allograft loss were identified as allocation of ECDs (hazard ratio 1.84 (95\% confidence interval 1.5 to 2.3); $P<0.001)$, presence of circulating DSA on the day of transplantation (3.00 (2.3 to 3.9); $\mathrm{P}<0.001$ ), and longer cold ischaemia time ( $>12 \mathrm{~h}$; 1.53 (1.1 to 2.1); $\mathrm{P}=0.011)$. Recipients of ECD kidneys with circulating DSA showed a 5.6-fold increased risk of graft loss compared with all other transplant therapies $(P<0.001)$. ECD allograft survival at seven years significantly improved with screening and transplantation in the absence of circulating DSA $(P<0.001)$ and with shorter $(<12 \mathrm{~h})$ cold ischaemia time $(P=0.030)$, respectively. This strategy achieved ECD graft survival comparable to that of patients receiving an SCD transplant overall, translating to a 544.6 allograft life years saved during the nine years of study inclusion time.

\section{CONCLUSIONS}

Circulating DSA and cold ischaemia time are the main independent determinants of outcome from ECD transplantation. Allocation policies to avoid DSA and reduction of cold ischaemia time to increase efficacy could promote wider implement of ECD transplantation in the context of organ shortage.

\section{Introduction}

Global population ageing has modified the landscape of chronic diseases with an increased prevalence of older patients with end stage renal failure. ${ }^{1}$ For such patients, kidney transplantation is the best approach, surpassing dialysis in terms of health related quality 
and quantity of life as well as cost effectiveness. ${ }^{2-4}$ Ageing of the general population reflects a constant and dramatic increase of the deceased donor age with accompanying burden of cardiovascular comorbidities, leading to the definition of an expanded criteria donor (ECD; that is, those aged $\geq 60$ years, or aged $50-59$ years with vascular comorbidities). ${ }^{5}$ About $30 \%$ and $47 \%$ of deceased kidney donors are ECDs in Europe and France, respectively. ${ }^{67}$ In the coming years, this dynamic will render ECD transplantation the main source for kidney transplants as well as other solid organ (heart, lung, and liver) transplants. ${ }^{67}$

But despite its potential, the use of ECD kidneys has evolved unevenly; only $16.6 \%$ of deceased donor transplants come from an ECD in the United States, and many harvested ECD organs are ultimately refused by transplant teams and discarded. ${ }^{8}$ These discrepancies reflect uncertainties regarding the prognosis and determinants associated with ECD transplants. The discrepancies also contribute to a major public health issue (with a high proportion (10-20\%) of viable organs being discarded worldwide) and to the deaths of patients with end stage renal disease waiting for a kidney transplant. ${ }^{9-11}$

Although ECD transplantation produces a benefit in recipient survival compared with dialysis, it is associated with an increased risk of graft loss compared with that associated with transplants from standard criteria donors (SCDs). ${ }^{51213}$ Currently, no comprehensive studies has addressed the primary independent determinants of ECD transplant outcomes by integrating deep donor and recipient phenotyping and large scale circulating anti-HLA (human leucocyte antigen) antibody characterisation. The need for such studies was identified by the International Banff Allograft Committee in $2013^{14}$ and public healthcare agencies ${ }^{15}$ to improve the use and allocation of ECD kidneys through a better understanding of long term outcomes. Thus, we conducted a large, prospective, population based study of extensively phenotyped kidney recipients to assess the distinct prognosis of ECD transplants and the factors that affect long term allograft survival.

\section{Methods}

\section{Study population}

We enrolled all 2763 consecutive patients who underwent kidney transplantation at four referral centres in France, including Necker Hospital in Paris ( $n=920)$, Saint-Louis Hospital in Paris ( $n=391)$, Foch Hospital in Suresnes $(n=597)$, and Toulouse University Hospital $(\mathrm{n}=855)$, between 1 January 2004 and 1 January 2011. Patients were followed up to 1 May 2014. We also included an additional external validation cohort comprising 4128 patients who underwent kidney transplantation at centres in Lyon, Montpellier, Nancy, and Nantes (France) between 1 January 2002 and 31 December 2011 (web appendix, supplementary methods).

The transplantation allocation system was identical for all centres, and it followed the rules of the French national agency for organ procurement (Agence de la Biomédecine). All transplants were compatible based on ABO blood group. Cytotoxicity cross matching of negative immunoglobulin $\mathrm{G} T$ cell and B cell complements was required for all the recipients. Post-transplant immunosuppression therapy and treatment of allograft rejection episodes were standardised between centres (web appendix, supplementary methods).

The institutional review boards of Necker, SaintLouis, Foch, and Toulouse Hospitals approved the study. The use of data from the external validation cohort was based on agreements between the centres that participate in the national database system (web appendix). All the recipients provided written informed consent.

\section{Patient involvement}

There was no patient involvement in this study. The selection of outcome measures was not informed by patients' priorities and experiences. No patients, service users, or lay people were involved in developing plans for participant recruitment and study conduct. The results of the study will be disseminated to participants through the physicians of each department. The participants are thanked in the acknowledgments.

\section{Clinical data}

We obtained clinical data on the donors and recipients in the development cohort (Necker, Saint-Louis, Foch, and Toulouse Hospitals) and the validation cohort (Lyon, Montpellier, Nancy, and Nantes) from two national registries: Données Informatiques Validées en Transplantation ${ }^{16}$ and Agence de la Biomédecine. ${ }^{17}$ Anonymised data from these registries were prospectively entered at a specific time point for each patient (on day 0 , and six months and one year after transplantation), and were updated annually thereafter (supplementary methods in the web appendix shows the clinical and biological parameters assessed). Data from Necker, Saint-Louis, Foch, and Toulouse Hospitals were retrieved from the database on 1 May 2014. Data from the validation cohort were retrieved on 20 June 2014.

\section{Definition of expanded criteria donor (ECD)}

Expanded criteria donors included all deceased donors aged 60 years and older, and those aged over 50-59 years with at least two of the following three conditions: cerebrovascular cause of death, serum creatinine greater than $1.5 \mathrm{mg} / \mathrm{dL}(132.6 \mu \mathrm{mol} / \mathrm{L})$, or a history of hypertension.

\section{Detection and characterisation of donor specific anti-HLA antibodies}

All the patients were tested for the presence of circulating donor specific anti-HLA antibodies (DSA) in banked serum samples (at the Jean Dausset Histocompatibility Laboratory) from Necker, Saint Louis, and Foch Hospitals and Toulouse Histocompatibility Laboratory at the time of transplantation (day 0 DSA). The presence of circulating DSA against HLA-A, HLA-B, HLA-Cw, HLA-DR, HLA-DQ, and HLA-DP was retrospectively determined using single antigen flow bead assays (One Lambda) on a Luminex platform. Beads 
with a normalised mean fluorescence intensity higher than 500 arbitrary units were judged positive, as previously described. ${ }^{18}$

HLA typing of the transplant recipients was performed using the Innolipa HLA typing kit (Innogenetics). Donor HLA typing for HLA-Cw and HLA-DP was only performed if recipients had circulating antiHLA-Cw or anti-HLA-DP (or both). Tissue typing was done using the microlymphocytotoxicity technique with One Lambda and tissue typing trays; traditional controls in molecular biology were included.

\section{Kidney allograft phenotypes}

In the principal cohort, kidney allograft biopsies were performed on the day of transplantation (preimplantation biopsies, $\mathrm{n}=1011$ ) and at one year after transplantation ( $\mathrm{n}=1743)$; biopsies were also taken from patients with acute allograft rejection during the first year after transplantation ( $\mathrm{n}=438)$. Renal biopsies were fixed in a solution of alcohol, formalin, and acetic acid, and subsequently embedded in paraffin. The biopsy sections ( $4 \mu \mathrm{m}$ thick) were stained with periodic acid-Schiff, Masson's trichrome, and haematoxylin and eosin. The graft biopsies were scored and graded by trained pathologists (JPD-V-H, MR, JV, and CG-F) according to the international Banff criteria for kidney allograft transplantation (web appendix). ${ }^{14}$

\section{Statistical analysis}

Continuous variables are described using means and standard deviations or medians and the interquartile range. We compared means and proportions between groups using Student's t test, analysis of variance (ANOVA; Mann-Whitney test for mean fluorescence intensity) or the $\chi^{2}$ test (or Fisher's exact test if appropriate). The kidney survival analysis was performed from the time of transplantation to a maximum follow-up of seven years with kidney graft loss as the event of interest, defined as the patient's return to dialysis. For the 204 (7.4\%) patients who died with a functioning graft, graft survival was censored at the time of death. ${ }^{19}$ Kidney allograft survival according to ECD and DSA status was plotted using Kaplan-Meier curves and compared using the log rank test.

Cox proportional hazards models were applied to quantify the hazard ratios and the $95 \%$ confidence intervals for kidney graft loss. We compared the graft life years between recipients of ECD allografts with and without baseline levels of DSA, and estimated the graft life years saved. To this end, we compared the area under the Kaplan-Meier curves between the two groups starting from a common time point, namely, the time of transplantation. ${ }^{20}$

The associations of donor, recipient, and transplant parameters as well as immunological factors with graft loss were first assessed in univariate regression analyses. The factors identified in these analyses were then included in a final multivariate model using stepwise backward selection. In the sensitivity analysis, a competing risks approach was applied to consider the potential competition of patient death with kidney allograft failure. We estimated cumulative incidence functions from competing risks data and compared the subdistribution for each cause across groups. We then assessed the effect of predictive factors (that is, ECD and DSA status) on the subdistribution of graft loss in a competing risks setting with death, by fitting the proportional subdistribution hazards regression model described in the Fine and Gray method. ${ }^{21}$ Propensity scores were used to test whether older recipient age affected allograft outcome and the associations identified in the primary analyses (web appendix). ${ }^{22}$

We confirmed the internal validity of the final model using a bootstrap procedure. A thousand datasets were generated from the resampling of the original dataset to estimate the biased corrected 95\% confidence intervals and the accelerated bootstrap hazard ratios. ${ }^{23}$

We used an additional external validation cohort comprising 4128 kidney transplant recipients. We first determined kidney allograft survival according to ECD and DSA status. We then tested in the validation cohort, the set of risk factors identified in the final multivariate Cox model of the development cohort. We used SAS 9.2 (SAS) and R (version 2.10.1, R Foundation for Statistical Computing) for the descriptive and survival analyses. All statistical tests were two sided, and $\mathrm{P}<0.05$ was considered significant.

\section{Results}

Baseline characteristics of recipients and donors

The main analysis included 2763 recipient patients. Median donor age was 52 years (interquartile range 40-61). A total of 1509 (54.6\%) donors were male, and $2370(85.8 \%)$ kidneys were from deceased donors, of whom 1323 (55.8\%) had died of cerebrovascular causes. All the deceased donors were brain dead. A total of 661 (24.4\%) donors had hypertension, and 142 (5.5\%) donors presented with diabetes mellitus. A total of 916 (33.2\%) kidneys fulfilled the criteria for ECD, whereas 1847 (66.8\%) were classified as SCD. Web figure 1 details the donor age distribution for the entire cohort. Cold ischaemia time is the period starting from retrieval and cold perfusion of an organ until reperfusion with warm blood in the recipient. The median cold ischaemia time was 16.5 hours (interquartile range 12-21). Of the 2763 recipients, $335(12.1 \%)$ had circulating DSA at the time of transplantation. The highest ranked DSA was HLA class II in $189(56.4 \%)$ patients and HLA class I in 146 patients (43.6\%). Mean fluorescence intensity of the immunodominant DSA had a median value of 2928 (interquartile range 1390-6835). Table 1 presents the baseline and immunological characteristics of the overall cohort according to ECD status.

Four distinct populations were identified on the basis of donor characteristics (SCD or ECD) and the presence or absence of circulating anti-HLA antibodies at the time of transplantation. These groups included: patients receiving SCD transplants without DSA (SCD/DSA-, $n=1622$ ); patients receiving SCD transplants with DSA (SCD/DSA+, $\mathrm{n}=225$ ); patients receiving ECD transplants without DSA (ECD/DSA-, $\mathrm{n}=806$ ); patients receiving ECD transplants with DSA 
Table 1 | Baseline characteristics according to donor status

\begin{tabular}{|c|c|c|c|c|}
\hline & $\begin{array}{l}\text { All patients } \\
(\mathrm{n}=2763)\end{array}$ & $\begin{array}{l}\text { Standard criteria } \\
\text { donors }(n=1847)\end{array}$ & $\begin{array}{l}\text { Expanded criteria } \\
\text { donors }(n=916)\end{array}$ & $\mathrm{P}^{*}$ \\
\hline \multicolumn{5}{|l|}{ Recipient characteristics } \\
\hline Age (years, mean (SD)) & $49.23(13.51)$ & $44.26(12.31)$ & $59.23(10.21)$ & $<0.001$ \\
\hline Male sex (no (\%)) & $1643(59.5)$ & $1105(59.8)$ & $538(58.7)$ & 0.582 \\
\hline \multicolumn{5}{|l|}{ Causes of end stage renal disease (no (\%)) } \\
\hline Glomerulonephritis & $767(27.8)$ & $561(30.4)$ & $206(22.5)$ & \multirow{4}{*}{$<0.001$} \\
\hline Diabetes & $264(9.5)$ & $171(9.2)$ & $93(10.1)$ & \\
\hline Hypertension & $212(7.7)$ & $129(7.0)$ & $83(9.1)$ & \\
\hline Other & $1520(55.0)$ & $986(53.4)$ & $534(58.3)$ & \\
\hline Time since onset of dialysis (years, mean (SD)) & $3.90(4.41)$ & $3.85(4.67)$ & $4.02(3.84)$ & 0.3451 \\
\hline \multicolumn{5}{|l|}{ Donor characteristics } \\
\hline Age (years, mean (SD)) & $50.31(15.98)$ & $42.34(12.51)$ & $66.37(8.29)$ & $<0.001$ \\
\hline \multicolumn{5}{|l|}{ Donor age (years, no (\%)) } \\
\hline $0-50$ & $1222(44.2)$ & $1222(66.2)$ & 0 & \multirow{3}{*}{$<0.00$} \\
\hline $50-60$ & $733(26.6)$ & $565(30.6)$ & $168(18.3)$ & \\
\hline$\geq 60$ & $808(29.2)$ & $60(3.2)$ & $748(81.7)$ & \\
\hline Male sex (no (\%)) & $1509(54.6)$ & $1056(57.2)$ & $453(49.5)$ & $<0.001$ \\
\hline Hypertension (no (\%))† & $661(24.4)$ & $167(9.2)$ & $494(55.0)$ & $<0.001$ \\
\hline Diabetes mellitus (no (\%)) $\ddagger$ & $142(5.5)$ & $40(2.3)$ & $102(11.8)$ & $<0.001$ \\
\hline Creatinine $>1.5 \mathrm{mg} / \mathrm{dL}$ (no (\%))§ & $270(9.9)$ & $169(9.3)$ & $101(11.0)$ & 0.150 \\
\hline \multicolumn{5}{|l|}{ Donor type (no (\%)) } \\
\hline Deceased donor & $2370(85.8)$ & $1454(78.7)$ & $916(100.0)$ & $<0.001$ \\
\hline Death from cerebrovascular disease & $1323(55.8)$ & $627(43.1)$ & $696(76.0)$ & $<0.001$ \\
\hline \multicolumn{5}{|l|}{ Transplant baseline characteristics } \\
\hline Graft rank >1 (no (\%)) & $413(15.0)$ & $288(15.6)$ & $125(13.7)$ & 0.177 \\
\hline \multicolumn{5}{|l|}{ Cold ischaemia time (no (\%))** } \\
\hline$<12 \mathrm{~h}$ & $674(24.5)$ & $594(32.3)$ & $80(8.8)$ & \multirow{3}{*}{$<0.001$} \\
\hline $12-24 \mathrm{~h}$ & $1560(56.7)$ & $910(49.5)$ & $650(71.0)$ & \\
\hline$\geq 24 \mathrm{~h}$ & $519(18.8)$ & $334(18.2)$ & $185(20.2)$ & \\
\hline 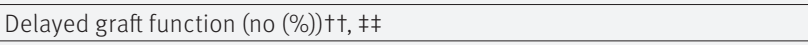 & $707(27.2)$ & $410(23.5)$ & $297(34.7)$ & $<0.001$ \\
\hline HLA A/B/DR mismatch (no, mean (SD))§§ & $3.12(1.43)$ & $3.07(1.47)$ & $3.24(1.33)$ & 0.005 \\
\hline Donor specific anti-HLA antibodies on day 0 (no (\%)) & $335(12.1)$ & $225(12.2)$ & $110(12.0)$ & 0.896 \\
\hline \multicolumn{5}{|l|}{ HLA class of donor specific anti-HLA antibodies (no) } \\
\hline HLA class I & 191 & 123 & 68 & 0.214 \\
\hline HLA class II & 245 & 166 & 79 & 0.704 \\
\hline HLA class I and II & 114 & 76 & 38 & 0.889 \\
\hline Donor specific anti-HLA antibodies (mean fluorescence intensity (median (IQR)) & $2928(1390-6835)$ & $2755(1360-6480)$ & $3005(1450-8369)$ & 0.099 \\
\hline Follow-up (years, median $(95 \% \mathrm{CI})$ ) & $5.54(5.42$ to 5.68$)$ & $5.72(5.54$ to 5.86$)$ & $5.24(5.02$ to 5.46$)$ & $<0.001$ \\
\hline \multicolumn{5}{|l|}{ Acute rejection in first year } \\
\hline Antibody mediated rejection (no (\%)) & $205(7.4)$ & $140(7.6)$ & $65(7.1)$ & 0.648 \\
\hline T cell mediated rejection (no (\%)) & $233(8.4)$ & $156(8.5)$ & $77(8.4)$ & 0.972 \\
\hline Death events (no (\%)) & $263(9.5)$ & $128(6.9)$ & $135(14.7)$ & $<0.001$ \\
\hline Graft loss (no (\%)) & $333(12.1)$ & $187(10.1)$ & $146(15.4)$ & $<0.001$ \\
\hline
\end{tabular}

Data are no (\%) of patients or events, mean (standard deviation), or median (interquartile range). SD=standard deviation; IQR=interquartile range; HLA=human leucocyte antigen.

${ }^{*} \chi^{2}$ tests used to compare proportions, and unpaired tests used to compare continuous variables.

total group numbers were: all patients $(\mathrm{n}=2709)$, standard criteria donors $(\mathrm{n}=1811)$, and expanded criteria donors $(\mathrm{n}=898)$.

抽al group numbers were: all patients $(\mathrm{n}=2604)$, standard criteria donors $(\mathrm{n}=1741)$, and expanded criteria donors $(\mathrm{n}=863)$.

$\S$ Total group numbers were: all patients $(n=2736)$, standard criteria donors $(n=1820)$, and expanded criteria donors $(n=916)$.

१Total group numbers were: all patients $(n=2370)$, standard criteria donors $(n=1454)$, and expanded criteria donors $(n=916)$.

**Total group numbers were: all patients $(n=2753)$, standard criteria donors $(n=1838)$, and expanded criteria donors $(n=915)$.

††Delayed graft function defined as use of dialysis in the first postoperative week.

$\neq \neq$ Total group numbers were: all patients $(n=2603)$, standard criteria donors $(n=1746)$, and expanded criteria donors $(n=857)$.

$\S \S$ Total group numbers were: all patients $(n=2700)$, standard criteria donors $(n=1844)$, and expanded criteria donors $(n=856)$.

(ECD/DSA+, $\mathrm{n}=110,12 \%$ of patients receiving ECD transplants). Table 2 details the baseline clinical and immunological characteristics of these four patient groups. Web figure 2 shows the histological characteristics of donor biopsies before implantation in the four patient groups.

Long term kidney allograft survival, injury phenotypes, and function

Median follow-up after transplantation was 5.54 years (interquartile range 4.01-7.42). Median follow-up times were 5.24 years (3.64-6.97) for ECD recipients and 5.72 years (4.16-7.82) for SCD recipients $(\mathrm{P}<0.001)$.

Figure 1A depicts kidney allograft survival according to ECD or SCD status. Overall, patients receiving ECD transplants had a higher rate of graft loss than those receiving SCD transplants (hazard ratio 1.87 (95\% confidence interval 1.50 to 2.32); log rank test $\mathrm{P}<0.001)$. After dividing patients into groups based on donor characteristics (SCD or ECD) and the presence of circulating DSA on day $0, E C D / D S A+$ recipients showed worse 


\begin{tabular}{|c|c|c|c|c|c|}
\hline & SCD/DSA $-(n=1622)$ & $S C D / D S A+(n=225)$ & $E C D / D S A-(n=806)$ & $\mathrm{ECD} / \mathrm{DSA}+(\mathrm{n}=110)$ & $P^{*}$ \\
\hline \multicolumn{6}{|l|}{ Recipient characteristics } \\
\hline Age (years, mean (SD)) & $44.20(12.20)$ & $44.76(11.65)$ & $59.60(9.98)$ & $56.53(11.41)$ & $<0.001$ \\
\hline Male sex (no (\%)) & $1001(61.7)$ & $104(46.2)$ & $489(60.7)$ & $49(44.6)$ & $<0.001$ \\
\hline Time since onset of dialysis (years, mean (SD)) & $3.56(4.28)$ & $5.93(6.45)$ & $3.74(3.58)$ & $6.01(4.91)$ & $<0.001$ \\
\hline \multicolumn{6}{|l|}{ Donor characteristics } \\
\hline Age (years, mean (SD)) & $42.25(12.50)$ & $43.01(12.61)$ & $66.43(8.24)$ & $65.95(8.67)$ & $<0.001$ \\
\hline \multicolumn{6}{|l|}{ Donor age (years, no (\%)) } \\
\hline $0-50$ & $1073(66.1)$ & $149(66.2)$ & 0 & 0 & \multirow{3}{*}{$<0.001$} \\
\hline $50-60$ & $496(30.6)$ & $69(30.7)$ & $147(18.2)$ & $21(19.1)$ & \\
\hline$\geq 60$ & $53(3.3)$ & $7(3.1)$ & $659(81.8)$ & $89(80.9)$ & \\
\hline Male sex (no (\%)) & $934(57.6)$ & $122(54.2)$ & $393(48.8)$ & $60(54.6)$ & 0.001 \\
\hline Hypertension (no (\%))† & $142(8.9)$ & $25(11.3)$ & $427(54.0)$ & $67(62.6)$ & $<0.001$ \\
\hline Diabetes mellitus (no (\%)) $\neq$ & $34(2.2)$ & $6(2.9)$ & $94(12.3)$ & $8(8.1)$ & $<0.001$ \\
\hline Creatinine $>1.5 \mathrm{mg} / \mathrm{dL}$ (no (\%))§ & $155(9.7)$ & $14(6.3)$ & $94(11.7)$ & $7(6.4)$ & 0.054 \\
\hline \multicolumn{6}{|l|}{ Donor type (no (\%)) } \\
\hline Deceased donor & $1273(78.5)$ & $181(80.4)$ & $806(100)$ & $110(100)$ & $<0.001$ \\
\hline Death from cerebrovascular diseaseी & $552(43.4)$ & $75(41.4)$ & $612(75.9)$ & $84(76.4)$ & $<0.001$ \\
\hline \multicolumn{6}{|l|}{ Transplant baseline characteristics } \\
\hline Graft rank >1 (no (\%)) & $193(11.9)$ & $95(42.2)$ & $85(10.6)$ & $40(36.4)$ & $<0.001$ \\
\hline \multicolumn{6}{|l|}{ Cold ischaemia time (no (\%)) $)^{\star \star}$} \\
\hline$<12 \mathrm{~h}$ & $544(33.7)$ & $50(22.5)$ & $70(8.7)$ & $10(9.1)$ & \multirow{3}{*}{$<0.001$} \\
\hline $12-24 \mathrm{~h}$ & $803(49.7)$ & $107(48.2)$ & $575(71.4)$ & $75(68.2)$ & \\
\hline$\geq 24 \mathrm{~h}$ & $269(16.6)$ & $65(29.3)$ & $160(19.9)$ & $25(22.7)$ & \\
\hline Delayed graft function (no (\%))†† & $343(22.4)$ & $67(30.9)$ & $256(33.9)$ & $41(40.2)$ & $<0.001$ \\
\hline HLA A/B/DR mismatch (no, mean (SD)) f & $3.07(1.47)$ & $3.09(1.48)$ & $3.21(1.32)$ & $3.44(1.35)$ & 0.015 \\
\hline \multicolumn{6}{|l|}{ HLA class of donor specific anti-HLA antibodies (no) on day 0} \\
\hline HLA class I & - & 123 & - & 68 & $0.214 \S \S$ \\
\hline HLA class II & - & 166 & - & 79 & $0.704 \S \S$ \\
\hline HLA class I and II & - & 76 & - & 38 & $0.889 \S \S$ \\
\hline $\begin{array}{l}\text { Donor specific anti-HLA antibodies (mean fluorescence } \\
\text { intensity (median (IQR)) }\end{array}$ & - & $2755(1360-6480)$ & - & $3005(1450-8369)$ & $<0.001$ \\
\hline Follow-up (years, median $(95 \% \mathrm{Cl})$ ) & $5.86(5.70$ to 6.12$)$ & 4.66 (4.34 to 4.92$)$ & $5.31(5.08$ to 5.54$)$ & 4.73 (4.24 to 5.19$)$ & $<0.001$ \\
\hline \multicolumn{6}{|l|}{ Acute rejection in the first year } \\
\hline Antibody mediated rejection (no (\%)) & $78(4.8)$ & $62(27.6)$ & $31(3.9)$ & $34(30.9)$ & $<0.001$ \\
\hline T cell mediated rejection (no (\%)) & $142(8.8)$ & $14(6.2)$ & $66(8.2)$ & $11(10.0)$ & 0.561 \\
\hline Death events (no (\%)) & $108(6.7)$ & $20(8.9)$ & $118(14.6)$ & $17(15.5)$ & $<0.001$ \\
\hline Graft loss (no (\%)) & $146(9.0)$ & $41(18.2)$ & $103(12.8)$ & $43(39.1)$ & $<0.001$ \\
\hline \multicolumn{6}{|c|}{ 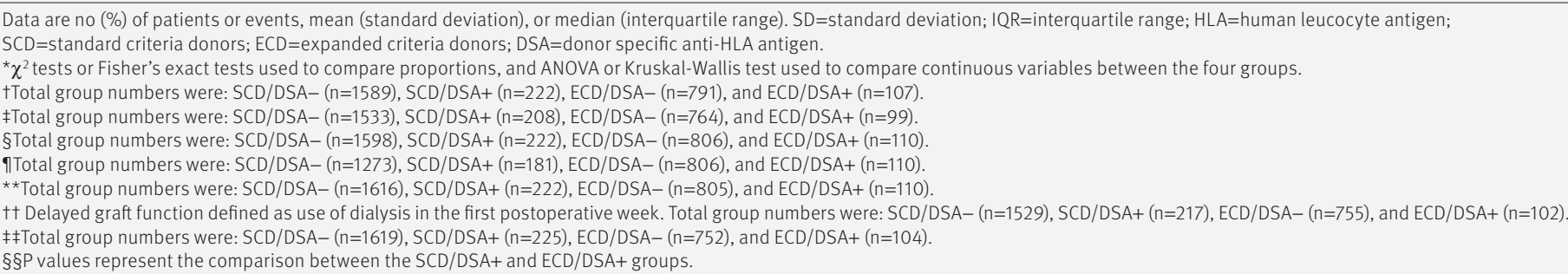 } \\
\hline
\end{tabular}

graft survival (44\%, seven years post-transplant) than ECD/DSA- (85\%), SCD/DSA+ (73\%), and SCD/DSA$(90 \%)$ recipients (log rank test, $\mathrm{P}<0.001)$. Antibody mediated rejection was the main cause of graft loss in the ECD/DSA+ group (58.1\% $v 14.6 \%$ in the ECD/DSAgroup; $\mathrm{P}<0.001)$.

Patients receiving ECD transplants without circulating DSA at the time of transplantation showed a $41 \%$ improvement in graft survival at seven years compared with those with circulating DSA at the time of transplantation $(\mathrm{P}<0.001$, fig $1 \mathrm{~B})$.

Patients receiving ECD transplants with circulating DSA had a 4.4-fold increased risk of graft loss compared with those receiving ECD transplants without circulat- ing DSA $(\mathrm{P}<0.001)$. ECD recipients with circulating DSA also had a 5.6-fold increased risk of graft loss compared with all other transplant therapies $(\mathrm{P}<0.001$; fig $1 \mathrm{~B})$.

Mean allograft life years for ECD/DSA+ recipients was 4.58 (standard deviation 0.22) compared with 9.53 (0.12) for ECD/DSA- recipients (mean gain survival of 4.95 years). Overall, we calculated that exclusively allocating ECD kidneys to patients without circulating DSA would have translated to a 544.6 allograft life years saved during the nine years of study inclusion time. Competing risks regression models confirmed that the differences in graft survival between the four groups were not affected by competition with patient death (web fig 3). 

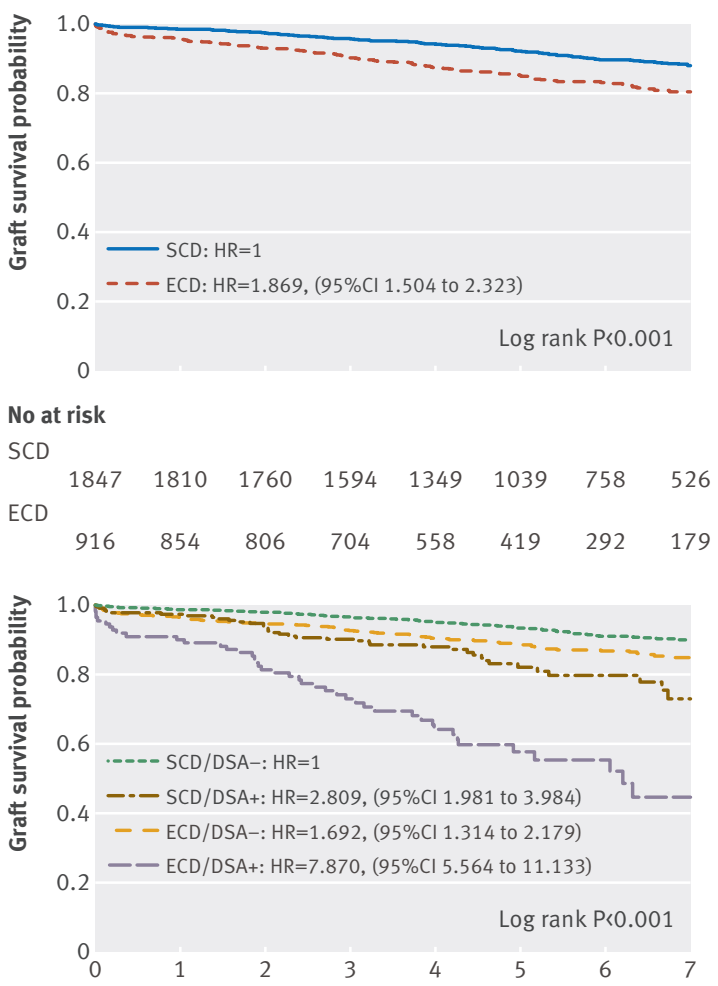

No at risk Time since transplantation (years)

SCD/DSA-

$\begin{array}{llllllll}1622 & 1591 & 1552 & 1419 & 1216 & 962 & 708 & 504\end{array}$

$\mathrm{SCD} / \mathrm{DSA}+$

$\begin{array}{llllllll}225 & 219 & 208 & 175 & 133 & 77 & 50 & 22\end{array}$

ECD/DSA-

$\begin{array}{llllllll}806 & 756 & 723 & 641 & 511 & 393 & 275 & 175\end{array}$

ECD/DSA+

$\begin{array}{llllllll}110 & 98 & 83 & 63 & 47 & 26 & 17 & 4\end{array}$

Kaplan-Meier curves of kidney allograft survival by (A) donor type and (B) presence of DSA on the day of transplantation. Panel A shows the classic approach to determine kidney allograft survival based on donor status (that is, SCD or ECD). Panel B integrates donor status with the presence or absence of DSA on the day of transplantation. $S C D=$ standard criteria donor; $E C D=$ expanded criteria donor; $D S A=$ donor specific anti-HLA antibodies; SCD/DSA-=patients receiving SCD transplants without DSA; SCD/DSA+=patients receiving SCD transplants with $D S A ; E C D / D S A$-=patients receiving ECD transplants without $D S A ; E C D / D S A+=$ patients receiving ECD transplants with DSA; HR=hazard ratio

An allograft evaluation at one year revealed that the patients receiving ECD transplants with circulating DSA had decreased kidney function compared with the other groups $(\mathrm{P}<0.001$, web fig 4$)$. Allograft biopsies at one year after transplantation showed that ECD/DSA+ recipients had increased microcirculation inflammation $(\mathrm{P}<0.001)$, arteriosclerosis $(\mathrm{P}=0.014)$, and transplant glomerulopathy lesions $(\mathrm{P}<0.001)$ compared with patients receiving ECD transplants without circulating DSA. ECD/DSA+ recipients also showed increased chronic allograft injury lesions (arteriosclerosis, arterial hyalinosis, and atrophy scarring) compared with the two groups of patients receiving SCD transplants $(\mathrm{P}<0.002$ for all comparisons, web fig 5).

\section{Determinants of kidney allograft loss}

Table 3 shows the associations of recipient and donor characteristics, transplant characteristics, and immunological parameters with graft loss. Table 4 shows the identified baseline independent predictors of graft loss. These predictors included: ECD status (hazard ratio 1.84 (95\% confidence interval 1.47 to 2.31 ); $\mathrm{P}<0.001$ ), presence of circulating DSA on day 0 (3.00 (2.27 to 3.94); $\mathrm{P}<0.001$ ), cold ischaemia time between 12 and 24 hours (1.46 (1.04 to 2.04); $\mathrm{P}=0.028)$; cold ischaemia time longer than 24 hours (1.73 (1.19 to 2.52); $\mathrm{P}=0.004)$; graft rank more than 1 (1.54 (1.17 to 2.04); $\mathrm{P}=0.002)$, and number of HLA A/B/DR mismatches (1.10 (1.01 to 1.18); $\mathrm{P}=0.022)$. The set of risk factors identified in the primary analysis remained unchanged when we included continuous cold ischaemia time in the final multivariate Cox model (web table 1).

When we performed the Cox analysis in the ECD group, the major determinants independently associated with graft failure were cold ischaemia between 12 and 24 hours (hazard ratio 2.49 (1.02 to 6.13); $\mathrm{P}=0.046$ ) and longer than 24 hours (3.77 (1.49 to 9.55); $\mathrm{P}=0.005$ ) and the presence of circulating DSA at day 0 (4.59 (3.18 to 6.61); $\mathrm{P}<0.001)$. Among patients receiving ECD transplants, those with a high level of DSA mean fluorescence intensity ( $\geq 3000)$ were at a higher risk of graft failure than those with a low level of DSA mean fluorescence intensity $(<3000)$ and those without circulating DSA $(\mathrm{P}<0.001$, web fig 6). We saw no difference between HLA class I and HLA class II groups regarding allograft survival (web fig 7). For the 1011 patients with an available biopsy from the day of transplantation, baseline histology parameters were not independently associated with the risk of long term kidney allograft loss (web table 2).

\section{Sensitivity analysis}

The robustness of our study was assessed using sensitivity analyses. The independent associations of DSA and cold ischaemia time with graft outcome were consistent after excluding living donors $(n=393,14.2 \%)$ from the final multivariate model (web table 3). DSA and cold ischaemia time remained independently associated with graft loss when allograft quality for age and cardiovascular comorbidities was assessed using the kidney donor risk index (KDRI) score ${ }^{24}$ instead of ECD status (web fig 8; KDRI adjusted DSA (hazard ratio 3.44 (95\% confidence interval 2.60 to 4.55), $\mathrm{P}<0.001)$; KDRI adjusted cold ischaemia time (1.81 (1.12 to 2.92), $\mathrm{P}=0.015$ ).

The use of non-a priori donor parameters (age, deceased donor, sex, comorbidities, and baseline renal function) instead of ECD criteria did not change the significant independent predictors of graft loss identified in the principal analyses (web table 4). When the final model was stratified by transplant centre, the set of risk factors identified in the principal analyses did not change (web table 5). Finally, in a matched sample with similar SCD and ECD recipient ages using the propensity score (web appendix, supplementary methods), the survival analysis produced similar results to those obtained in the primary analyses (web fig 9). 


\begin{tabular}{|c|c|c|c|}
\hline & $\begin{array}{l}\text { No of patients/ } \\
\text { events }\end{array}$ & Hazard ratio $(95 \% \mathrm{Cl})$ & $P$ \\
\hline \multicolumn{4}{|l|}{ Baseline recipient characteristics } \\
\hline Age (per 1 year increment) & $2763 / 333$ & $1.000(0.992$ to 1.008$)$ & 0.973 \\
\hline \multicolumn{4}{|l|}{ Sex } \\
\hline Female & $1120 / 131$ & 1 & \multirow{2}{*}{0.648} \\
\hline Male & $1643 / 202$ & $1.053(0.845$ to 1.311$)$ & \\
\hline \multicolumn{4}{|l|}{ Baseline donor characteristics } \\
\hline Age (per 1 year increment) & $2763 / 333$ & 1.014 (1.007 to 1.021$)$ & $<0.001$ \\
\hline \multicolumn{4}{|l|}{ Sex } \\
\hline Female & $1254 / 163$ & 1 & \multirow{2}{*}{0.164} \\
\hline Male & $1509 / 170$ & 0.858 (0.692 to 1.064$)$ & \\
\hline \multicolumn{4}{|l|}{ Donor type } \\
\hline Living related & $393 / 25$ & 1 & \multirow{3}{*}{0.001} \\
\hline Death from cardiovascular disease & $1323 / 184$ & 2.136 (1.406 to 3.245$)$ & \\
\hline Other cause of death & $1047 / 124$ & 1.716 (1.116 to 2.638$)$ & \\
\hline \multicolumn{4}{|l|}{ Deceased donor } \\
\hline No & $393 / 25$ & 1 & \multirow{2}{*}{0.001} \\
\hline Yes & $2370 / 308$ & 1.945 (1.294 to 2.925) & \\
\hline \multicolumn{4}{|l|}{ Hypertension } \\
\hline No & $2048 / 225$ & 1 & \multirow{2}{*}{$<0.001$} \\
\hline Yes & $661 / 102$ & 1.647 (1.302 to 2.082$)$ & \\
\hline \multicolumn{4}{|l|}{ Diabetes mellitus } \\
\hline No & $2462 / 292$ & 1 & \multirow{2}{*}{0.050} \\
\hline Yes & $142 / 22$ & $1.543(1.000$ to 2.380$)$ & \\
\hline \multicolumn{4}{|l|}{ Creatinine } \\
\hline$<1.5 \mathrm{mg} / \mathrm{dL}$ & $2466 / 291$ & 1 & \multirow{2}{*}{0.900} \\
\hline$\geq 1.5 \mathrm{mg} / \mathrm{dL}$ & $270 / 40$ & $0.964(0.547$ to 1.700$)$ & \\
\hline \multicolumn{4}{|l|}{ Expanded criteria donor } \\
\hline No & $1847 / 187$ & 1 & \multirow{2}{*}{$<0.001$} \\
\hline Yes & $916 / 146$ & 1.869 (1.504 to 2.323$)$ & \\
\hline
\end{tabular}

\section{Baseline transplant characteristics}

\begin{tabular}{|c|c|c|c|}
\hline \multicolumn{3}{|l|}{ Graft rank } & \multirow{3}{*}{$<0.001$} \\
\hline 1 & $2350 / 249$ & 1 & \\
\hline$>1$ & $413 / 84$ & 2.174 (1.697 to 2.785$)$ & \\
\hline \multicolumn{4}{|l|}{ Cold ischaemia time } \\
\hline$<12 \mathrm{~h}$ & $674 / 44$ & 1 & \multirow{3}{*}{$<0.001$} \\
\hline $12-24 \mathrm{~h}$ & $1560 / 203$ & 1.877 (1.355 to 2.601$)$ & \\
\hline$\geq 24 \mathrm{~h}$ & $519 / 85$ & $2.392(1.662$ to 3.443$)$ & \\
\hline \multicolumn{4}{|c|}{ Baseline immunological characteristics } \\
\hline No of HLA A/B/DR mismatches & $2700 / 326$ & $1.080(1.001$ to 1.116$)$ & 0.047 \\
\hline \multicolumn{4}{|l|}{ Anti-HLA DSA on day 0} \\
\hline No & $2428 / 249$ & 1 & \multirow{2}{*}{$<0.001$} \\
\hline Yes & $335 / 84$ & $3.440(2.676$ to 4.421$)$ & \\
\hline
\end{tabular}

DSA=donor specific anti-HLA antibodies; HLA=human leucocyte antigen.

\section{Internal and external validation}

A bootstrapping procedure with 1000 samples from the original dataset confirmed the internal validity and robustness of the final model (biased corrected 95\% confidence intervals and accelerated bootstrap hazard ratios, table 4). Baseline characteristics of the external validation cohort (4128 patients) were compared with those of the principal cohort in web table 6 . In this cohort, the HLA sensitisation level at the time of transplantation was estimated using cytotoxic panel reactive antibodies (PRAs) or calculated PRAs without identifying specific anti-HLA antibodies. The Kaplan-Meier estimate of graft survival confirmed that patients receiving ECD transplants with circulating DSA on the day of transplantation had a higher risk of graft loss at seven years than those without circulating DSA and the two groups of patients receiving SCD transplants (web fig 10). The multivariate Cox model of the external cohort confirmed the independent predictors of allograft failure identified in the principal analyses (web table 7).

\section{Discussion}

\section{Principal findings}

In a large, prospective, population based study of extensively phenotyped recipients of kidney transplants, we assessed the long term allograft survival and determinants of ECD prognosis using an integrative epidemiological approach that combined clinical donor and recipient data, histopathology, and immunological characteristics. We identified a group of people with poor prognosis-that is, patients receiving ECD transplants with circulating DSA. This group had a 4.4-fold increased risk of graft loss compared with patients receiving ECD transplants without circulating DSA, and had a 5.6-fold increased risk of graft loss compared with all other transplant therapies. When patients receiving ECD transplants were extensively screened and transplanted without DSA, graft survival increased by $41 \%$, reaching a seven year rate of allograft survival of $85 \%$ (versus 90\% in patients receiving SCD transplants without circulating DSA).

\section{Implication of results}

In the past decade, the constant rise in donor age in the organ pool has generated new challenges for optimising organ use, especially when considering the characteristics of an older organ in terms of response to injury after transplantation. This problem has raised concerns beyond kidney transplantation-extending to lung, liver, and heart transplants-and has become a top priority for allocation agencies.

ECD kidney transplantation has evolved unequally between countries. ECD programmes have been largely promoted and implemented in Europe and France and represent about half of deceased donor kidney transplantations. ${ }^{6}$ However, in the USA, a high proportion of ECD organs are discarded, and ECD organs represent only $16.6 \%$ of deceased donor allografts. ${ }^{8}$ Data from US scientific registries indicate that over a six year period, 5139 (41\%) of 12536 ECD kidneys were not transplanted.

ECD therapy has demonstrated benefits over dialysis in terms of patient survival ${ }^{13}$ but has diminished long term allograft survival. ${ }^{25}$ Therefore, the improvement of ECD outcomes and specific allocation is critical for the transplant community and allocation agencies as well as for general practitioners dealing with population ageing and the burden of chronic renal disease.

Currently, an important gap exists regarding the independent determinants of ECD outcome and methods for improving ECD allograft survival in the context of allograft assessments, which include contemporary tools for evaluating circulating anti-HLA antibodies. Although circulating DSA are known to impair allograft outcomes, their specific effect, the amplitude of their effect, and their independency from other relevant 


\begin{tabular}{|c|c|c|c|c|}
\hline & No of patient/events & Hazard ratio $(95 \% \mathrm{Cl})$ & $P$ & $\begin{array}{l}\text { Internal validation } \\
\text { hazard ratio } 95 \% \mathrm{Cl}, \mathrm{BCA}\end{array}$ \\
\hline No & $1835 / 187$ & 1 & \multirow{2}{*}{$<0.001$} & - \\
\hline Yes & $855 / 138$ & $1.842(1.467$ to 2.311$)$ & & (1.463 to 2.275$)$ \\
\hline $12-24 \mathrm{~h}$ & $1514 / 198$ & $1.457(1.042$ to 2.039$)$ & \multirow[t]{2}{*}{0.017} & (1.042 to 2.093) \\
\hline$\geq 24 \mathrm{~h}$ & $506 / 83$ & 1.727 (1.185 to 2.517$)$ & & (1.195 to 2.506$)$ \\
\hline \multicolumn{5}{|l|}{ Graft rank } \\
\hline 1 & $2278 / 241$ & 1 & \multirow{2}{*}{0.002} & - \\
\hline$>1$ & $412 / 84$ & 1.544 (1.168 to 2.042$)$ & & (1.129 to 2.046$)$ \\
\hline Yes & $326 / 84$ & 2.988 (2.265 to 3.941$)$ & $<0.001$ & (2.198 to 3.940$)$ \\
\hline \multicolumn{5}{|c|}{$\begin{array}{l}\text { *The final multivariate Cox model was obtained by entering risk factors from the univariate model that achieved P } \leq 0.10 \text { as the thresholds in a single } \\
\text { multivariate proportional hazards model. The final multivariate model was adjusted for the following parameters: deceased donor, donor diabetes, } \\
\text { expanded criteria donor, graft rank, cold ischaemia time, number of HLA A/B/DR mismatches, and circulating DSA. The test for potential interactions } \\
\text { between graft rank, ECD status, cold ischaemia time, HLA mismatch, and circulating DSA generated P>0.10 for all the interactions. } \\
\text { DSA=donor specific anti-HLA antibodies; BCA=bias corrected and accelerated bootstrap; HLA=human leucocyte antigen. }\end{array}$} \\
\hline
\end{tabular}

predictors have not been specifically analysed in the recipients of ECD transplants. ${ }^{186-29}$ The structure of the existing registries-the United Network of Organ Sharing in the USA, ${ }^{30}$ Eurotransplant, ${ }^{31} 32$ the Organ Procurement Agency in Spain, ${ }^{33}$ and the French National Agency for Organ Procurement ${ }^{17}$-does not specifically integrate the whole spectrum of important determinants that might affect ECD prognosis.

In the present study, we used a prospective, extensively phenotyped cohort with a detailed and integrative strategy of allograft parameter analysis, and demonstrated that ECD kidney recipients with circulating DSA show a dramatic acceleration in antibody mediated injury and a poor outcome. Another important independent and strong contributor of ECD survival was longer cold ischaemia time $(>12 \mathrm{~h})$, which was associated with a 2.6-fold decrease in seven year allograft survival. Increased cold ischaemia time predisposes to delayed graft function, but its independent role in long term allograft outcome is still debated..$^{3435}$ Our data suggest that ECD kidneys might be vulnerable to the detrimental effects of cold ischaemia time on allograft survival.

Finally, we demonstrated that preimplantation biopsy assessments did not have independent and additional predictive ability for long term ECD allograft outcome when considering cold ischaemia time and immunological parameters (that is, circulating DSA). Given this result and the fact that preimplantation biopsies increase cold ischaemia time, the current practice of discarding ECD kidneys based on preimplantation biopsy results might not be the best approach for decision making in the context of ECD transplants, as recently suggested in small preliminary studies. ${ }^{936}$

Our results support the implementation of active ECD specific allocation policies for avoiding DSA, decreasing cold ischaemia time, and performing adequate recipient matching. In the present study, exclusive allo- cation of ECD kidneys to patients without circulating DSA would have translated to a 544.6 allograft life years saved during the nine years of study inclusion time.

\section{AUTHOR AFFILIATIONS}

${ }^{1}$ Paris Translational Research Centre for Organ Transplantation, INSERM, UMR-S970, Paris Descartes University, 75015 Paris, France

2Department of Nephrology and Organ Transplantation, Rangueil, Toulouse, France

${ }^{3}$ INSERM U1043, Purpan, Toulouse

4Paul Sabatier University, Toulouse

5DIVAT (Données Informatiques VAlidées en Transplantation Network), France

${ }^{6}$ Department of Nephrology and Kidney Transplantation, Saint-Louis Hospital, Assistance Publique Hôpitaux de Paris

${ }^{7}$ Department of Kidney Transplantation, Necker Hospital, Assistance Publique Hôpitaux de Paris, Sorbonne Paris

${ }^{8}$ Department of Pathology, Necker Hospital, Paris

${ }^{9}$ Department of Renal Physiology, Georges Pompidou European Hospital, Paris

${ }^{10}$ Department of Pathology, Saint-Louis Hospital, Paris

${ }^{11}$ Molecular Immunogenetics Laboratory, EA 3034, Faculty of Medicine Purpan, IFR150 (INSERM), Toulouse, France

12Department of Immunology, Rangueil Hospital, Toulouse

${ }^{13}$ Department of Pathology, Rangueil, Toulouse

${ }^{14}$ Department of Nephrology and Kidney Transplantation, Montpellier, France

15Department of Nephrology and Kidney Transplantation, Groupement Hospitalier, Hôpital Edouard Herriot, Lyon, France ${ }^{16}$ Centaure Network, France

17Department of Nephrology and Kidney Transplantation, Nantes, France

${ }^{18}$ Department of Nephrology and Kidney Transplantation, Nancy, France

${ }^{19}$ Department of Nephrology and Kidney Transplantation, Foch Hospital, Suresnes, Paris

${ }^{20}$ Department of Cardiology, Georges Pompidou European Hospital, Paris

We thank all patients included in the present study.

Contributors: OA, NK, DV, XJ, CaL, and AL have shared first authorship. OA, NK, CaL, DG, FM, ChL, XJ, and AL designed the study. OA, AL, CaL, DV, JPD-V-H, NK, LR, NC, MR, JV, FM, MD, GM, EM, MG, and CG-F collected the data. Data analysis was performed by OA, DW, AL, CaL, 
NK, J-PE, and XJ. OA, NK, DW, DE, ChL, XJ, CaL, and AL wrote the final report. All authors contributed to both protocol and final report. $A L$ is guarantor. All the authors had full access to all of the data and can take responsibility for the integrity of the data and the accuracy of the data analysis.

Funding: There was no specific funding for this study.

Competing interests: All authors have completed the ICMJE uniform disclosure form at www.icmje.org/coi disclosure.pdf and declare: no support from any organisation for the submitted work, no financial relationships with any organisations that might have an interest in the submitted work in the submitted work in the previous three years, no other relationships or activities that could appear to have influenced the submitted work.

Ethical approval: The research protocol was reviewed and approved by the local institutional review boards.

Patient consent: Codes were used to ensure the strict donor and recipient anonymity and blind assay. Informed consent was obtained from participants at time of transplantation.

Data sharing: No additional data available.

The guarantor affirms that the manuscript is an honest, accurate, and transparent account of the study being reported; that no important aspects of the study have been omitted; and that any discrepancies from the study as planned have been explained.

This is an Open Access article distributed in accordance with the Creative Commons Attribution Non Commercial (CC BY-NC 4.0) license, which permits others to distribute, remix, adapt, build upon this work non-commercially, and license their derivative works on different terms, provided the original work is properly cited and the use is non-commercial. See: http://creativecommons.org/licenses/ by-nc/4.0/.

1 Réseau Epidémiologie et Information en Néphrologie. Annual report. 2013. www.socnephrologie.org/PDF/enephro/registres/ rapport_2013/rapport_rein2013.pdf.

2 Evans RW, Manninen DL, Garrison LP Jr, et al. The quality of life of patients with end-stage renal disease. N Engl / Med 1985;312:553-9.

3 Port FK, Wolfe RA, Mauger EA, et al. Comparison of survival probabilities for dialysis patients vs cadaveric renal transplant recipients. JAMA 1993;270:1339-43.

4 Rana A, Gruessner A, Agopian VG, et al. Survival benefit of solid-organ transplant in the United States. JAMA Surg 2015;150:252-9.

5 Port FK, Bragg-Gresham IL, Metzger RA, et al. Donor characteristics associated with reduced graft survival: an approach to expanding the pool of kidney donors. Transplantation 2002;74:1281-6.

6 Eurotransplant. Annual report. 2013. https://www.eurotransplant.org/ $\mathrm{cms} /$ mediaobject.php?file=AR20135.pdf

7 L'Agence de la Biomédecine. Annual report. 2013. www.agencebiomedecine.fr/rapport-annuel-2013.

8 Organ Procurement and Transplantation Network/Scientific Registry of Transplant Recipients. Annual report. 2012. srtr.transplant.hrsa. gov/annual_reports/2012/pdf/2012_SRTR_ADR.pdf.

9 Sung RS, Christensen LL, Leichtman AB, et al. Determinants of discard of expanded criteria donor kidneys: impact of biopsy and machine perfusion. Am J Transplant 2008;8:783-92.

10 Cecka JM, Gritsch HA. Why are nearly half of expanded criteria donor (ECD) kidneys not transplanted? Am / Transplant 2008;8:735-6.

11 Tanriover B, Mohan S, Cohen DJ, et al. Kidneys at higher risk of discard: expanding the role of dual kidney transplantation. Am J Transplant 2014:14:404-15.

12 Metzger RA, Delmonico FL, Feng S, et al. Expanded criteria donors for kidney transplantation. Am J Transplant 2003;3 Suppl 4:114-25.
13 Merion RM, Ashby VB, Wolfe RA, et al. Deceased-donor characteristics and the survival benefit of kidney transplantation. JAMA 2005;294:2726-33.

14 Haas M. Sis B, Racusen LC, et al. Banff 2013 meeting report: inclusion of c4d-negative antibody-mediated rejection and antibodyassociated arterial lesions. Am J Transplant 2014;14:272-83.

15 Sack K. In discarding of kidneys, system reveals its flaws. New York Times 2012 Sept 19

16 Données Informatiques Validées en Transplantation (DIVAT) software. Homepage. www.divat.fr/.

17 Agence de la Biomédecine. Homepage. www.sipg.sante.fr/portail/.

18 Lefaucheur C, Loupy A, Hill GS, et al. Preexisting donor-specific HLA antibodies predict outcome in kidney transplantation. J Am SoC Nephrol 2010;21:1398-406.

19 Lamb KE, Lodhi S, Meier-Kriesche HU. Long-term renal allograf survival in the United States: a critical reappraisal. Am J Transplant $2011 ; 11: 450-62$

20 Lee ET. Statistical methods for survival data analysis/2nd edition, John Wiley \& Sons, 1992.

21 Fine JP, Gray RJ. A proportional hazards model for the subdistribution of a competing Risk. J Am Stat Assoc 1999:94:496-509.

22 D'Agostino RB Jr. Propensity score methods for bias reduction in the comparison of a treatment to a non-randomized control group. Stat Med 1998;17:2265-81.

23 Efron B. Bootstrap methods: another look at the jackknife. Ann Stats 1979;7:1-26

24 Rao PS, Schaubel DE, Guidinger MK, et al. A comprehensive risk quantification score for deceased donor kidneys: the kidney donor risk index. Transplantation 2009;88:231-6.

25 Ojo AO, Hanson JA, Meier-Kriesche H, et al. Survival in recipients of marginal cadaveric donor kidneys compared with other recipients and wait-listed transplant candidates. J Am Soc Nephrol 2001;12:589-97.

26 Nankivell BJ, Alexander SI. Rejection of the kidney allograft. New Engl I Med 2010;363:1451-62.

27 Sellares J, de Freitas DG, Mengel M, et al. Understanding the causes of kidney transplant failure: the dominant role of antibody-mediated rejection and nonadherence. Am J Transplant 2012;12:388-99.

28 Nankivell BJ, Kuypers DR. Diagnosis and prevention of chronic kidney allograft loss. Lancet 2011;378:1428-37.

29 Gaston RS, Cecka JM, Kasiske BL, et al. Evidence for antibodymediated injury as a major determinant of late kidney allograft failure. Transplantation 2010:90:68-74.

30 Tuttle-Newhall JE, Krishnan SM, Levy MF, et al. Organ donation and utilization in the United States: 1998-2007. Am J Transplant 2009;9:879-93.

31 Frei U, Noeldeke J, Machold-Fabrizii V, et al. Prospective age-matching in elderly kidney transplant recipients-a 5-year analysis of the Eurotransplant Senior Program. Am J Transplant 2008;8:50-7.

32 Smits JM, Persijn GG, van Houwelingen HC, et al. Evaluation of the Eurotransplant Senior Program. The results of the first year. Am J Transplant 2002;2:664-70.

33 Ledo-Garcia E, Riera L, Passas J, et al. Spanish consensus document for acceptance and rejection of kidneys from expanded criteria donors. Clin Transplant 2014;28:1155-66

34 Kayler LK, Srinivas TR, Schold JD. Influence of CIT-induced DGF on kidney transplant outcomes. Am / Transplant 2011;11:2657-64.

35 Debout A, Foucher Y, Trebern-Launay K, et al. Each additional hour of cold ischemia time significantly increases the risk of graft failure and mortality following renal transplantation. Kidney Int 2015:87:343-9.

36 Azancot MA Moreso F, Salcedo M, et al. The reproducibility and predictive value on outcome of renal biopsies from expanded criteria donors. Kidney Int 2014:85:1161-8.

(C) BMI Publishing Group Ltd 2015

Web appendix: Supplementary material 